\title{
Xpektrin, un simulador de espectros fácil de usar y am- pliamente distribuible para radiología general
}

\author{
Camilo Alberto de la Barra Olate', Marianela Andrea Hervias Jara².
}

1. Centro de Imagenología Hospital Clínico de la Universidad de Chile. Santiago, Chile.

2. Departamento de Tecnología Médica de la Universidad de Chile. Santiago, Chile.

Xpektrin, an easy to use and highly distributable X-Ray Spectra Simulator in General Radiography.

Resumen: Se presenta una aplicación basada en Microsoft Excel llamada Xpektrin para el cálculo de dosis en radiología general. La aplicación permite simular espectros de rayos $X$ en radiología general utilizando el modelo TASMICS a partir de mediciones del kerma en aire $\left(K_{\text {air }}\right)$ y de la capa Hemirreductora (HVL). Tiene implementado el cálculo de magnitudes radiométricas y dosimétricas, como el kerma en aire en la superficie de entrada $\left(K_{e}\right)$ y la dosis en piel $\left(D_{\text {skin }}\right)$, en función de la elección arbitraria de los factores de exposición, el tipo y grosor de filtro, la distancia foco-piel y el tamaño de campo. Xpektrin fue validado con la herramienta computacional SPEKTR 3.0, utilizando mediciones de dosis y de HVL de tubos de rayos $X$ de tres recintos hospitalarios. Se encontró buena correlación en ambas aplicaciones entre las mediciones experimentales y los valores calculados de HVL y con coeficientes de Pearson $R^{2} \geq 0.99$ en todos los casos. Sin embargo, se obtuvo mejor concordancia con los valores experimentales de HVL con Xpektrin (mediana de diferencias $-0.43 \%$, -0.04\% y 0.01\%) que con SPEKTR 3.0 (mediana de diferencias $-3.31 \%$, $0.10 \%$ y $-7.85 \%)$, en particular para el tubo con mayor filtración. Xpektrin está optimizada para ser utilizada en los departamentos de radiología para la determinación de dosis de pacientes individuales en función de los parámetros utilizados durante la exposición, por lo que puede ser utilizada como parte de un sistema de registro dosimétrico o como apoyo para el establecimiento de niveles de referencia para diagnóstico (NRD), siendo particularmente útil en servicios con equipos sin registros automáticos de dosis. Además, debido a sus características de simulador, puede ser útil como herramienta pedagógica. El uso de Excel permite que sea altamente distribuible y fácil de usar, sin necesidad de conocimientos de programación. Palabras clave: Dosis de radiación, Espectro de rayos $X$, Niveles de Referencia para Diagnóstico, Radiología general, TASMICS.

Abstract: Xpektrin, an easy to use and highly distributable X-Ray Spectra Simulator in General Radiography. An application based on Microsoft Excel called Xpektrin is presented for dose calculation in general radiology. The application was developed to simulate $X$-ray spectra in general radiography using the TASMICS model. Using as inputs air kerma $\left(K_{\text {air }}\right)$ and Half-value layer $(H V L)$ measurements, Xpektrin allows the calculation of several radiometric and dosimetric quantities, such as the entrance surface air kerma $\left(K_{e}\right)$ and the skin dose $\left(D_{\text {skin }}\right)$, depending on the exposure factors, filter material type, filter thickness, focus-skin distance and field size.

Xpektrin was validated against the Matlab toolkit SPEKTR 3.0, using dose and HVL measurements of $X$-ray tubes from three different hospitals. It was found good correlation in both applications between the experimental measurements and the calculated HVL and $K_{\text {air }}$ values with Pearson coefficients $R^{2} \geq 0.99$ in all cases. However, experimental and calculated HVL have better agreement with Xpektrin (median percent difference $-0.43 \%,-0.04 \%$ and $0.01 \%$ ) than SPEKTR 3.0 (median percent difference $-3.31 \%, 0.10 \%$ and $-7.85 \%)$, particularly for the tube with greater filtration thickness. Xpektrin is optimized to be used in radiology departments for patient dose determination depending on the exposure parameters and may be used as part of a dosimetric record system or as a support for the determination of Diagnostic Reference Levels, which may be useful when no automatic dose records are available. In addition, due to its simulator characteristics, it can be useful as a pedagogical tool. Using Excel allows Xpektrin to be highly distributable and easy to use, without the need for programming skills.

Keywords: Diagnostic Reference Levels, General radiography, Radiation dose, TASMICS, X-ray spectrum. 
de la Barra $C$, et al. Xpektrin, un simulador de espectros fácil de usar y ampliamente distribuible para radiología general. Rev Chil Radiol 2019; 25(3): 94-102.

${ }^{\star}$ Correo electrónico: Camilo de la Barra / cdelabarra@hcuch.cl. Marianela Hervias / mherviasj@uchile.cl

Trabajo enviado el 06 de mayo de 2019. Aceptado para publicación el 14 de agosto de 2019.

\section{Introducción}

En radiología general, la generación de información diagnóstica adecuada con exposición de radiación mínima para pacientes y el personal, depende en gran medida del funcionamiento del equipo y de su uso por el operador. Por este motivo, se han estandarizado varios controles al equipo que requieren diversos tipos de mediciones, como el estudio del rendimiento del tubo (dosis absorbida en aire sin retro dispersión por unidad de carga) y la medición de la capa Hemirreductora (Half Value layer, HVL, por sus siglas en inglés) ${ }^{(1,2)}$. Por lo general, estos parámetros son determinados a través de la medición del kerma en aire $\left(K_{\text {air }}\right)$. Adicionalmente, debido a la amplia distribución de dosis que puede tener un mismo procedimiento, la Comisión Internacional de Protección Radiológica (ICRP) propone el uso de niveles de referencia para diagnóstico (NRD) para mejorar la optimización de los procesos, donde, por ejemplo, si los NRD locales resultan ser inferiores a los NRD nacionales, se debiese dar mayor énfasis a la optimización de la calidad de imagen que a la reducción de dosis de radiación ${ }^{(3)}$. En radiología simple se recomienda determinar los NRD para las exploraciones de tórax, abdomen, pelvis y columna, así como también para las exploraciones de cráneo, debido a que involucran órganos sensibles en el rostro. Cuando no se dispone de medios para la medición del producto kerma-área, la International Atomic Energy Agency (IAEA) recomienda la medición del kerma en aire en superficie del tejido $\left(K_{\text {air, }}\right)^{(1)}$. Su determinación requiere un fantoma para incluir la contribución de la retro dispersión, aunque puede prescindirse al realizar el producto entre el $K_{\text {air }}$ y un factor de retro dispersión apropiado (BSF).

Para obtener valores de $K_{\text {air }}$ en diferentes escenarios sin necesidad de realizar múltiples mediciones, se utilizan modelos empíricos que permiten calcular $K_{\text {air }} u$ otras magnitudes de manera indirecta en función de los factores de exposición ( $\mathrm{kV}$ y mAs). Este tipo de metodología puede complementarse con modelos espectrales de producción de rayos $\mathrm{X}$ para aumentar la versatilidad y aumentar la cantidad de magnitudes radiométricas y dosimétricas que se pueden calcular. Los modelos espectrales existentes están dirigidos a radiología simple, intervencionismo, mamografía, tomografía computada y radioterapia de ortovoltaje, donde las aplicaciones incluyen cálculos dosimétricos, análisis de calidad de imagen, estudio de imágenes de doble energía, modelación de filtros y apoyo en la realización de simulaciones Monte Carlo, entre otros $^{(4,5,6,7)}$. Uno de los más utilizados es el modelo empírico TASMIPS desarrollado por Boone en $1997^{(8,9)}$, y actualizado en 2014 por Hernandez en base a simulaciones Monte Carlo, de nombre TASMICS ${ }^{(10)}$.

En este contexto, se desarrolló Xpektrin, una aplicación basada en Microsoft Excel que permite calibrar los espectros TASMICS en función de mediciones dosimétricas de un tubo de rayos $\mathrm{X}$ particular para luego realizar variados cálculos dosimétricos.

Xpektrin está optimizado para ser utilizado en departamentos de radiología para la determinación de dosis de pacientes individuales en función de los parámetros utilizados durante la exposición $(\mathrm{kV}, \mathrm{mAs}$, colimación y distancia). Xpektrin podría utilizarse como parte de un sistema de registro dosimétrico o como apoyo para el establecimiento de niveles de referencia para diagnóstico (NRD), siendo particularmente útil en servicios con equipos sin registros automáticos de dosis. El uso de Excel permite que sea altamente distribuible y fácil de usar, sin necesidad de conocimientos de programación. Además, debido a sus características de simulador, puede ser útil como herramienta pedagógica.

\section{Material y métodos \\ Descripción de la calibración de los espectros}

Xpektrin está basado en la planilla de cálculo original de Hernandez y Boone ${ }^{(10)}$, de donde se han extraído los espectros TASMICS de 50 a 150 $\mathrm{kV}$ y varios coeficientes de atenuación lineales de materiales comúnmente utilizados en la filtración de tubos de rayos $X$. Xpektrin determina la filtración de aluminio requerida para que los espectros TASMICS reproduzcan la calidad del haz del tubo analizado, en términos de HVL de aluminio. Se implementó un algoritmo iterativo programado en lenguaje VBA (Visual Basic for Applications) que determina el grosor de aluminio que minimiza la diferencia absoluta entre los valores de HVL calculados en los espectros filtrados $\mathrm{y}$ las mediciones experimentales de cada $\mathrm{kV}$ :

$$
\sum_{i=1}^{n}\left|H V L_{\text {calc }}\left(k V_{i}\right)-H V L_{\text {exp }}\left(k V_{i}\right)\right|
$$


La filtración de un grosor de aluminio se calcula con la ley de atenuación exponencial: $\Phi(E)=\Phi_{0}$ $(E) \cdot \exp \left(-d_{\bullet} \mu_{A l}(E)\right)$. El cálculo del HVL se realiza con un método similar al empleado en la planilla de cálculo de Hernández y Boone, determinando la transmisión dada por diferentes grosores de aluminio (entre 0 a 20 $\mathrm{mm}$ ) y luego realizando una interpolación semilogarítmica con un ajuste polinomial de tercer grado. Se realiza una segunda estimación del HVL disminuyendo el rango de búsqueda alrededor del valor encontrado previamente para mejorar la precisión del cálculo.

Una vez determinada la calidad de los espectros en función del HVL de aluminio, se prosigue a normalizar la fluencia de acuerdo al siguiente factor de proporcionalidad $^{(10)}$.

$$
C(k V)=\frac{\left(K_{\text {air }}\right)_{\exp }(k V)}{\left(K_{\text {air }}\right)_{\text {calc }}(k V)}
$$

Donde $\left(K_{\text {air }}\right)_{\text {exp }}$ es el kerma en aire experimental normalizado por mAs y $\left(K_{\text {air }}\right)_{\text {calc }}$ es el kerma en aire calculado a partir de los espectros TASMICS filtrados con el grosor de aluminio determinado previamente. Para obtener valores de $\left(K_{\text {air }}\right)_{\text {exp }}$ en todos los voltajes de tubo, se realiza una interpolación polinomial de segundo grado a los valores experimentales (curva de rendimiento). Los valores de $\left(K_{\text {air }}\right)_{c a l c}$ se estiman a partir del kerma electrónico de los espectros filtrados ${ }^{(11,12)}$ :

$$
\left(K_{\text {air }}\right)_{\text {calc }}=\sum_{1 \mathrm{keV}}^{150 \mathrm{keV}} \Phi_{\mathrm{E}} \cdot E \cdot\left(\frac{\mu_{\text {en }}(E)}{\rho}\right)_{\text {Aire }} \cdot \Delta E
$$

Donde $\left(\left(\mu_{e n}(E)\right) / \rho\right)_{\text {Aire }}$ es el coeficiente másico de absorción de energía del aire en función de la energía, obtenido de la base de datos del NIST ${ }^{(13)}$, mediante interpolaciones log-log con splines cúbicos para el rango de energía utilizado.

\section{Estimación de dosis en piel}

Se utiliza la metodología propuesta en Benmakhlouf et al. 2011 ${ }^{(14)}$ para la obtención de la dosis absorbida en la superficie de agua a partir del kerma en aire calculado:

$$
K_{\text {air }} \underset{\times \mathrm{BSF}}{\longrightarrow} K_{\text {air,e }} \underset{\times\left(\frac{\mu_{\text {en }}}{\rho}\right)_{\text {Agua_Aire }}}{\longrightarrow} K_{\text {agua }}=D_{\text {agua }}
$$

Donde $\left(\mu_{\text {en }} / \rho\right)_{\text {Aqua Aire }}$ es el factor que incorpora la retro dispersión de un fantoma de agua y es el factor que permite la conversión a kerma en agua. Estos factores se encuentran tabulados en función del $\mathrm{kV}$, $\mathrm{HVL}$, coeficiente de homogeneidad $\left(H V L_{1} / H V L_{2}\right)$ y tamaño de campo. Para la obtención de valores en función de las características espectrales del haz analizado, Xpektrin realiza interpolaciones sucesivas, primero interpolaciones con un polinomio de grado 2 para el HVL y luego interpolaciones lineales para el voltaje de tubo y tamaño de campo. Adicionalmente se considera la influencia del espesor del fantoma de agua para el cálculo del factor de retro dispersión según lo descrito en Benmakhlouf et al. 2013 ${ }^{(15)}$. Esta metodología permite extrapolar valores de BSFy $\left(\mu_{\text {en }}\right.$

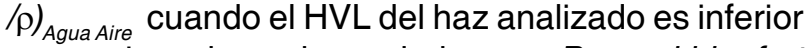
o superior a los valores dados por Benmakhlouf et al. 2011, aunque se despliega una advertencia en la hoja de cálculo cuando esto sucede.

El valor de la dosis en piel se estima directamente con el valor de la dosis en la superficie del fantoma de agua en la superficie $\left(D_{\text {piel }} \sim D_{\text {agua }}\right)$. Finalmente, para incluir la dependencia con los factores de exposición, se considera que la dosis es proporcional a la carga de tubo ( $m A s)$ y al inverso del cuadrado de la distan$\mathrm{cia}^{(16)}$, en un rango de $50 \mathrm{~cm}$ a $300 \mathrm{~cm}$ desde el foco.

\section{Prueba de la aplicación}

Se realizaron mediciones de dosis absorbida, $H V L$ y filtración para tres tubos de rayos $\mathrm{X}$ de radiodiagnóstico de servicios hospitalarios diferentes de marcas General Electric, Siemens y Varian, respectivamente. Se utilizó el detector de estado sólido Radcal AGMS-D+, asumiendo que, para el rango de energía utilizado, la dosis absorbida en aire es equivalente al kerma en aire debido a que las pérdidas radiativas son despreciables en condiciones de equilibrio electrónico. Cada medición se repitió tres veces y se calculó el valor promedio, abarcando el rango disponible del voltaje de cada tubo. Los valores experimentales de $K_{\text {air }}$ se normalizaron con respecto al mAs utilizado en cada disparo.

Las mediciones se utilizaron para calibrar los espectros TASMICS en Xpektrin para luego calcular la filtración inherente y los valores de y HVL en función del voltaje de tubo.

Los resultados se contrastaron con la aplicación SPEKTR 3.0 ${ }^{(17)}$, que es un conjunto gratuito de funciones de Matlab (TheMathworks, Natick, MA) para el análisis de espectros de rayos $\mathrm{X}$ basado en el modelo TASMICS. Se utilizaron los espectros determinados con la función spektrSpectrum(kV, [0 0],'TASMICS', 1) junto con la función spektrTuner para calcular la filtración inherente compuesta de aluminio y tungsteno requerida para reproducir los valores experimentales de $K_{\text {air }}$ para cada $\mathrm{kV}$ de las mediciones. A los grosores de los filtros de aluminio y tungsteno determinados 
por SPEKTR 3.0 se les sumó $1.6 \mathrm{~mm}$ de aluminio para considerar la filtración inherente de los espectros TASMIPS y se promediaron los valores de cada $\mathrm{kV}$. Los valores de HVL y $K_{\text {air }}$ se calcularon con las funciones incluidas en SPEKTR 3.0.

Se determinó el índice de correlación de Pearson y la pendiente de la recta ajustada entre los valores experimentales y los valores de HVL calculados en Xpektrin y SPEKTR 3.0 de cada tubo.

\section{Resultados}

Las mediciones de los tubos 1 (Figura 1) y 2 abarcan desde 40 a $150 \mathrm{kV}$, mientras que el tubo 3 tiene un rango de uso acotado entre 55 a $125 \mathrm{kV}$. Todas las mediciones se utilizaron como inputs en Xpektrin para calibrar los espectros, a diferencia de SPEKTR 3.0 donde se utilizaron solo los valores de $K_{\text {air }}$ Los valores de la filtración calculada en Xpektrin, el valor promedio de las filtraciones calculadas en SPEKTR 3.0 y el promedio de las mediciones experimentales se muestran en la tabla 1. Los valores de y $H V L$ determinados en Xpektrin y SPEKTR 3.0, junto con las mediciones experimentales se muestran en las figuras 2 y 3 , respectivamente. La correlación entre los valores experimentales y calculados de HVL para Xpektrin y SPEKTR 3.0 resultaron tener coeficientes de Pearson $R^{2} \leq 0.99$ en todos los casos, los valores de las pendientes de los ajustes se muestran en la tabla 2.

Tabla 1. Filtración de los tubos analizados.

\begin{tabular}{|llccc|}
\hline \multicolumn{2}{|c}{$\begin{array}{c}\text { Xpektrin } \\
(\mathbf{m m ~ A l )}\end{array}$} & $(\mathbf{m m ~ A l )}$ & $\begin{array}{c}\text { SPEKTR 3.0 } \\
(\boldsymbol{\mu} \mathbf{m ~ W})\end{array}$ & $\begin{array}{c}\text { AGMS } \\
(\mathbf{m m ~ A l})\end{array}$ \\
\hline Tubo 1 & 3.25 & $3.21 \pm 0.10$ & $0.48 \pm 0.14$ & $3.22 \pm 0.16$ \\
Tubo 2 & 3.86 & $4.03 \pm 0.24$ & $0.23 \pm 0.12$ & $3.95 \pm 0.10$ \\
Tubo 3 & 6.04 & $5.28 \pm 0.20$ & $0.24 \pm 0.14$ & $6.12 \pm 0.05$ \\
\hline
\end{tabular}

Tabla 2. Ajuste lineal entre HVL experimental y HVL calculado.

\begin{tabular}{|lll|}
\hline & Xpektrin & SPEKTR 3.0 \\
\hline Tubo 1 & $\mathrm{y}=1.009 \mathrm{x}$ & $\mathrm{y}=0.983 \mathrm{x}$ \\
Tubo 2 & $\mathrm{y}=1.006 \mathrm{x}$ & $\mathrm{y}=1.007 \mathrm{x}$ \\
Tubo 3 & $\mathrm{y}=0.999 \mathrm{x}$ & $\mathrm{y}=0.923 \mathrm{x}$ \\
\hline
\end{tabular}

Se obtuvo una mediana de diferencias entre los valores calculados de HVL y los valores experimentales de $-0.43 \%,-0.04 \%$ y $0.01 \%$ en Xpektrin y de $-3.31 \%,-0.10 \%$ y $-7.85 \%$ en SPEKTR 3.0 (Figura 4). Por otro lado, en las diferencias con los valores experimentales de $K_{\text {air }}$ se obtuvo una mediana de diferencias de $0.04 \%,-0.06 \%$ y $-1.08 \%$ en Xpektrin y $-0.55 \%,-1.71 \%$ y $-1.33 \%$ en SPEKTR 3.0 (Figura 5).

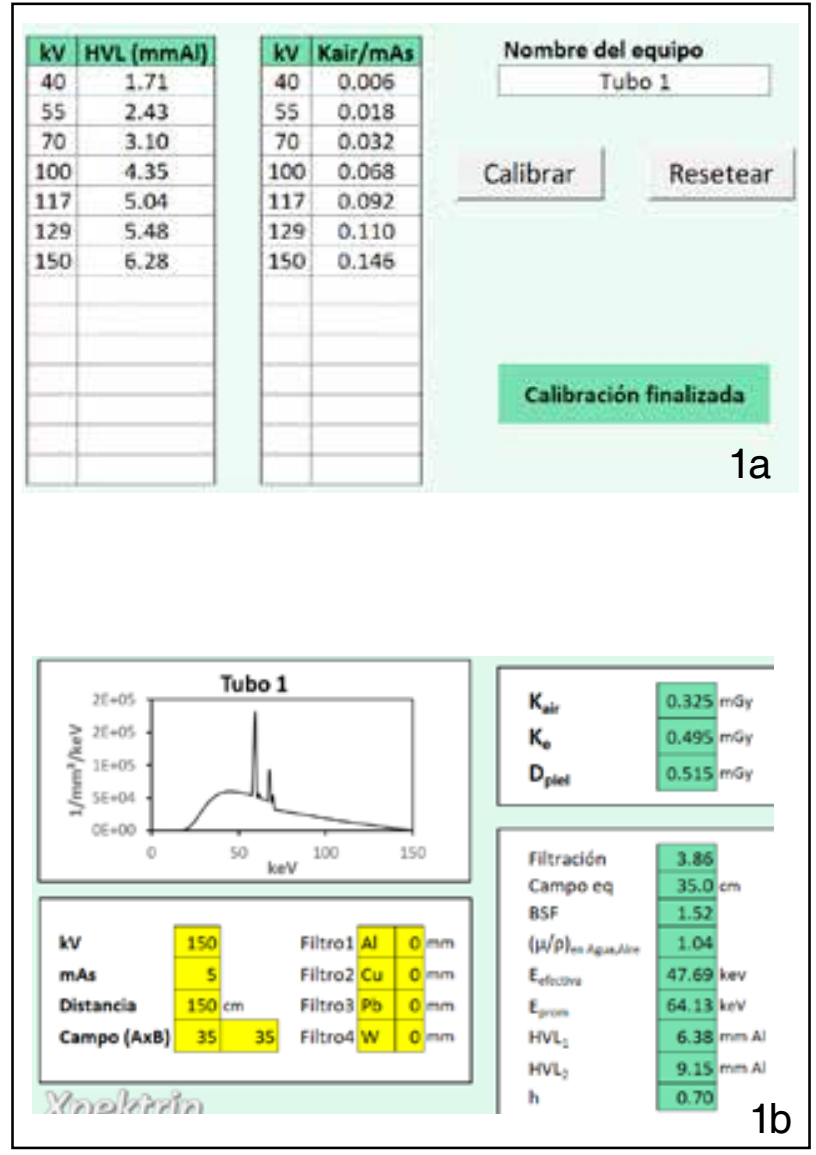

Figura 1: Capturas de pantalla de Xpektrin.

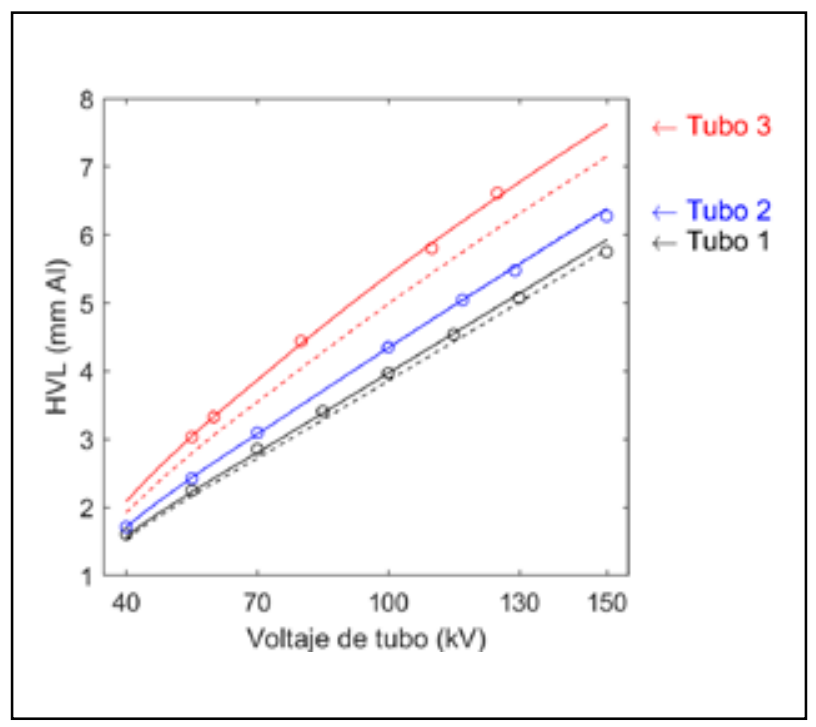

Figura 2: Comparación entre HVL calculado en Xpektrin (líneas continuas), HVL calculado en SPEKTR 3.0 (líneas con guiones) y mediciones experimentales de HVL (círculos) para los tubos analizados. 


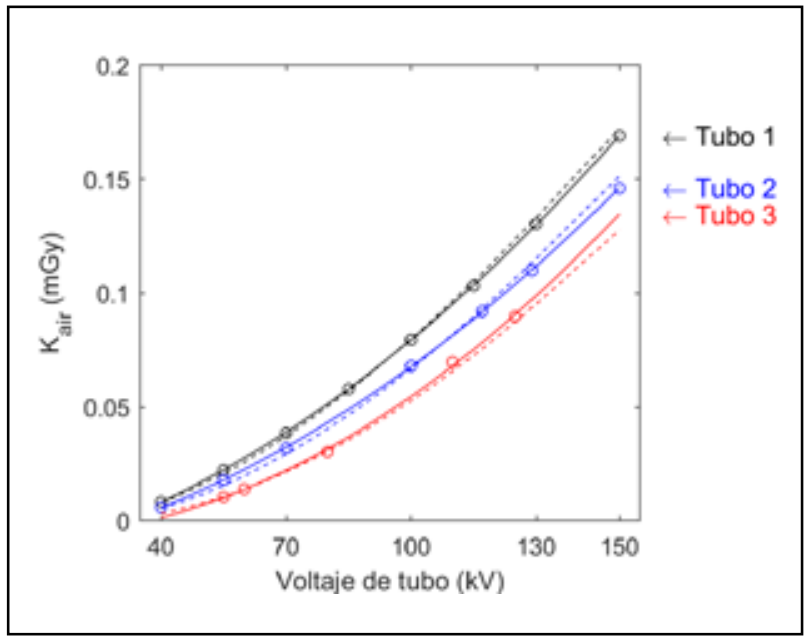

Figura 3: Comparación entre $K_{\text {air }}$ calculado en Xpektrin (líneas continuas), calculado en SPEKTR 3.0 (líneas con guiones) y mediciones experimentales de (círculos) para los tubos analizados
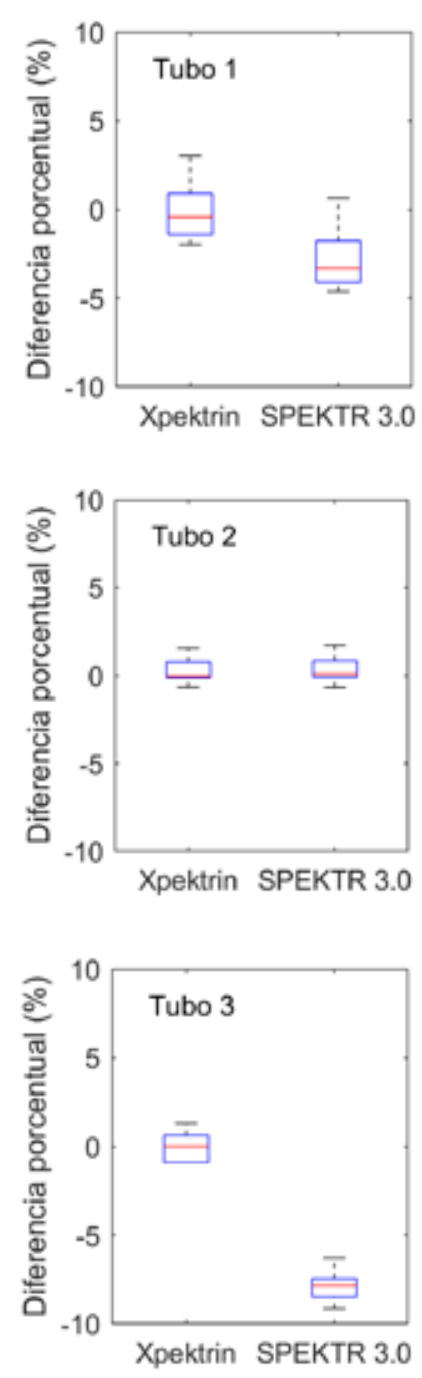

$4 b$

$4 a$

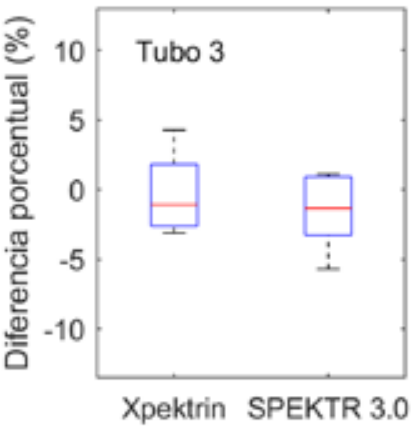

$5 c$

Figura 5: Diferencia de los valores calculados de con los valores experimentales.

\section{Discusión}

Los valores calculados con Xpektrin tuvieron buena concordancia con los valores experimentales para todos los tubos analizados, con una mediana de diferencias cercana a $0 \%$ en todos los casos. La máxima diferencia encontrada en los valores calculados de HVL por Xpektrin es de $3.0 \%$ para el tubo

Figura 4: Diferencia de los valores calculados de HVL con los valores experimentales. 
1, mientras que la máxima diferencia encontrada para los valores de $K_{\text {air }}$ es de $4.3 \%$ para el tubo 3 . La IAEA recomienda un nivel de precisión de $20 \%$ para la realización de dosimetría en exploraciones de baja dosis y de 7\% para exploraciones donde se esperan efectos deterministas ${ }^{(1)}$, lo que valida el uso de Xpektrin en radiología general.

La calibración de SPEKTR 3.0 se realiza de manera independiente para cada $\mathrm{kV}$, obteniéndose en cada caso un grosor de filtración. En este trabajo se realizó un promedio entre todos estos grosores de filtración obtenidos por SPEKTR 3.0 para cada $\mathrm{kV}$ con el fin de poder compararlo con los resultados de Xpektrin para cada tubo analizado. La filtración de SPEKTR 3.0 calculada de esta manera se compone fundamentalmente de aluminio, con grosores de tungsteno prácticamente despreciables; por ejemplo, al agregar un filtro de $0.5 \mu \mathrm{m}$ de tungsteno a los $3.25 \mathrm{~mm}$ de aluminio del tubo 1 , se produce una disminución del $K_{\text {air }}$ de $1.9 \%$ a $50 \mathrm{kV}$ y de $0.8 \%$ a $150 \mathrm{kV}$, mientras que el $H V L$ aumenta $0.7 \%$ y $0.6 \%$ para 50 y $150 \mathrm{kV}$ respectivamente, según cálculos realizados en Xpektrin. Se encontró buena concordancia entre los grosores de aluminio calculados en SPEKTR 3.0 y los valores experimentales para los tubos 1 y 2 ; sin embargo, el espesor de aluminio calculado para el tubo 3 difiere en $14 \%$. Este resultado concuerda con la gran diferencia del HVL calculado en SPEKTR 3.0 con respecto a las mediciones experimentales del tubo 3 . La dificultad en reproducir apropiadamente este espectro con alta filtración podría deberse a que SPEKTR 3.0 utiliza una única normalización basada en el rendimiento de tubo de los espectros TASMIPS ${ }^{(17)}$ que tienen baja

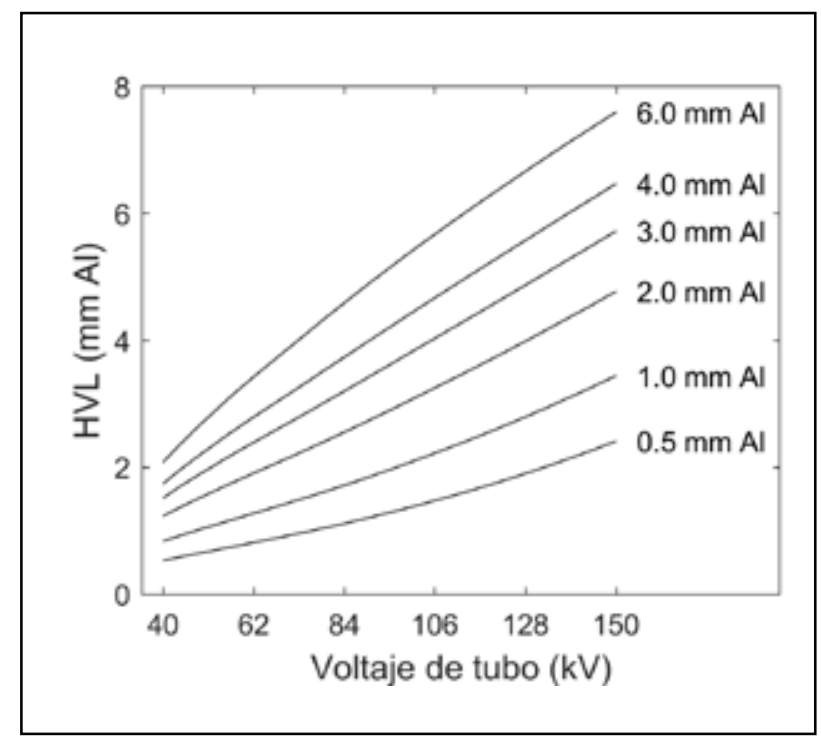

Figura 6: Valores de HVL en función del voltaje de tubo para diferentes filtraciones de los espectros TASMICS calculados en Xpektrin. filtración total (1.6 mm Al). Por el contrario, la normalización utilizada en Xpektrin se realiza para cada tubo analizado, en función del ajuste polinomial realizado a los valores experimentales de $K_{\text {air }}$, lo que permite reproducir los rendimientos de cada tubo, evitando la dependencia de la filtración total ${ }^{(10)}$.

El grosor calculado de aluminio es una buena aproximación de la filtración total del tubo de rayos $X$ debido a que los espectros TASMICS están diseñados con una filtración muy baja (0.08 $\mathrm{mm}$ de berilio). El algoritmo utilizado reduce directamente la diferencia entre los $H V L$ calculados y los valores experimentales logrando una metodología más versátil capaz de reproducir las características espectrales de tubos con alta filtración, como la del tubo 3. Sin embargo, si gran parte de la filtración del tubo correspondiera a materiales diferentes al aluminio o si existiese una metalización del tubo acentuada ${ }^{(18)}$, la reproducción de la calidad del haz en términos de HVL de aluminio por parte de Xpektrin podría disminuir su precisión. Gráficamente, la calibración de la calidad se basa en encontrar la filtración que produzca la curva de HVL en función del voltaje de tubo que mejor se acomode a las mediciones experimentales (Figura 6). Si bien un único valor experimental basta para la elección de una curva de $H V L$, varias mediciones permiten ponderar el comportamiento en diferentes rangos de $\mathrm{kV}$. El valor calculado de la filtración puede variar levemente en función del número de mediciones utilizadas para calibrar Xpektrin; por ejemplo, si se emplea únicamente el valor de $H V L$ medido en $85 \mathrm{kV}$ para calibrar el tubo 1, la filtración inherente cambia de 3,25 a 3,30 (Figura 7).

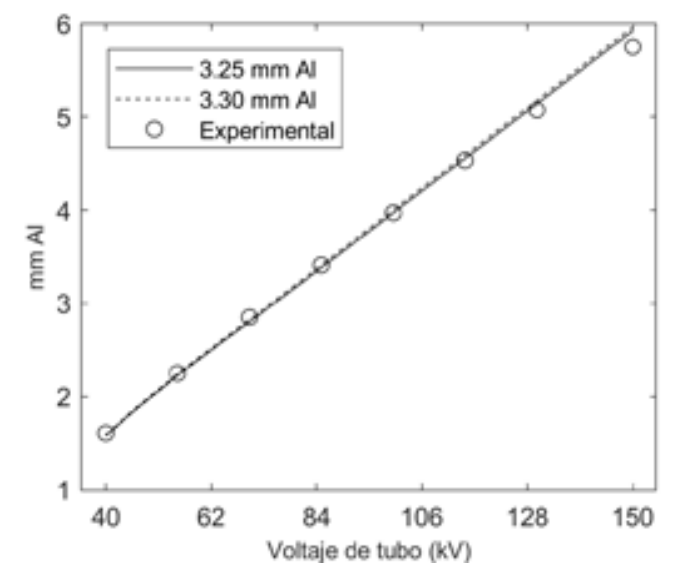

Figura 7: La curva de HVL con filtro $3.25 \mathrm{~mm}$ de Al se obtuvo al calibrar Xpektrin con todas las mediciones disponibles de HVL. La curva con $3.30 \mathrm{~mm}$ de Al se obtuvo calibrando Xpektrin solo con el valor de HVL medido en $85 \mathrm{kV}$. 
Xpektrin utiliza el $H V L$ y el kV para realizar interpolaciones lineales de los factores de Benmakhlouf, siendo estos dos parámetros apropiados para caracterizar la calidad del haz y realizar cálculos dosimétri$\cos ^{(19)}$. De esta manera, se logra gran versatilidad en la determinación del kerma en aire en la superficie de entrada y de la dosis absorbida en piel incluso para haces endurecidos con filtros adicionales, dentro del rango abarcado por los factores de Benmakhlouf. La capacidad de estimar la dosis absorbida con buena precisión para múltiples combinaciones de kV, mAs, distancia, tamaño de campo y filtración añadida, permite determinar la dosimetría de prácticamente todas las exploraciones realizadas en un servicio de manera retrospectiva o prospectiva, tanto para un paciente estándar, como para pacientes que requieran mayores niveles de exposición.

Utilizando Xpektrin se determinó $K_{\text {air }}, K_{e}$ y $D_{\text {piel }}$ para las radiografías de Tórax PA y Columna lumbar
AP con los factores de exposición típicos utilizados en cada hospital (Tabla 3). Los valores de $K_{e}$ calculados son similares a los niveles de referencia de dosis descritos en la literatura ${ }^{(20,21,22,23,24,25,26,27)}$ (Tabla 4). Si se posee un registro de los parámetros de exposición para exploraciones individuales de una muestra representativa de pacientes, Xpektrin podría ayudar a estimar los indicadores dosimétricos de cada una con el fin de proveer la estadística necesaria para la obtención de los valores típicos de NRD de una institución. Este tipo de metodología es de uso frecuente en la determinación de NDR en radiología general, aunque por lo general se suelen utilizar modelos empíricos para la obtención de los valores dosimétricos. Xpektrin permite la caracterización espectral de los haces utilizados y calcula a ella los valores dosimétricos y los factores de retro dispersión lo que podría mejorar la exactitud. Un seguimiento de valores típicos de NDR de una institución podría

Tabla 3. Magnitudes dosimétricas para radiografías determinadas con Xpektrin a partir de condiciones estándar para cada servicio

\begin{tabular}{|c|c|c|c|c|c|c|c|c|}
\hline & & \multicolumn{4}{|c|}{ Parámetros de exposición } & \multicolumn{3}{|c|}{ Magnitudes dosimétricas } \\
\hline & & $\mathrm{kV}$ & mAs & $\begin{array}{l}\text { DFP } \\
\text { (cm) }\end{array}$ & $\begin{array}{c}\text { Campo } \\
\text { (cm) }\end{array}$ & $\begin{array}{l}\text { Kair } \\
\text { (mGy) }\end{array}$ & $\begin{array}{c}\text { Ke } \\
\text { (mGy) }\end{array}$ & $\begin{array}{c}\text { Dosis en piel } \\
\text { (mGy) }\end{array}$ \\
\hline \multirow[t]{3}{*}{ Tórax PA } & Tubo 1 & 120 & 2.5 & 150 & $35 \times 35$ & 0.12 & 0.19 & 0.19 \\
\hline & Tubo 2 & 150 & 3 & 150 & $35 \times 35$ & 0.19 & 0.30 & 0.31 \\
\hline & Tubo 3 & 125 & 2.5 & 150 & $35 \times 35$ & 0.10 & 0.15 & 0.16 \\
\hline \multirow[t]{3}{*}{ Lumbar AP } & Tubo 1 & 70 & 63 & 80 & $24 \times 30$ & 3.81 & 5.23 & 5.33 \\
\hline & Tubo 2 & 80 & 70 & 80 & $24 \times 30$ & 4.69 & 6.69 & 6.83 \\
\hline & Tubo 3 & 85 & 90 & 80 & $24 \times 30$ & 5.12 & 7.63 & 7.82 \\
\hline
\end{tabular}

Tabla 4. Niveles de dosis de referencia de ESD para las radiografías de tórax PA y columna lumbar AP.

\begin{tabular}{|lccccccc|}
\hline & $\begin{array}{c}\text { TECDOC } \\
1646\end{array}$ & $\begin{array}{c}\text { European } \\
\text { Commission } \\
2010\end{array}$ & $\begin{array}{c}\text { Aroua } \\
1998,2014\end{array}$ & $\begin{array}{c}\text { Compagnone } \\
2005\end{array}$ & $\begin{array}{c}\text { Hart } \\
2009\end{array}$ & $\begin{array}{c}\text { Johnston } \\
2000\end{array}$ & $\begin{array}{c}\text { Roch } \\
\end{array}$ \\
& & & & & & & \\
\hline Tórax PA & $0,28 \mathrm{mGy}$ & $0.3 \mathrm{mGy}$ & $0.2 \mathrm{mGy}$ & $0.3 \mathrm{mGy}$ & $0.14 \mathrm{mGy}$ & $0.219 \mathrm{mGy}$ & $0.3 \mathrm{mGy}$ \\
Lumbar AP & $4,38 \mathrm{mGy}$ & $10 \mathrm{mGy}$ & $8.7 \mathrm{mGy}$ & $3.8 \mathrm{mGy}$ & $5.1 \mathrm{mGy}$ & $6.47 \mathrm{mGy}$ & $10 \mathrm{mGy}$ \\
\hline
\end{tabular}


ayudar a identificar la existencia de valores mayores o menores de los anticipados indicando cuándo es necesario iniciar una investigación de las prácticas locales con el interés de regresar al nivel de radiación utilizado previamente o justificar clínicamente el aumento o disminución de la dosis ${ }^{(3)}$.

Xpektrin está diseñado para ser utilizado en un contexto clínico en los servicios de radiología general con una interfaz gráfica sencilla que facilita su uso sin requerir conocimientos especializados en el manejo de Excel ni de programación. Adicionalmente, se pueden agregar fácilmente funcionalidades a la planilla Excel dependiendo de los requerimientos del usuario. Si bien Xpektrin requiere mediciones adicionales de HVL en comparación con SPEKTR 3.0, éstas podrían obtenerse de controles de calidad del equipo, sumado a que la creciente disponibilidad de multímetros en los servicios clínicos facilita las mediciones. En este contexto, Xpektrin debiese utilizarse en equipos con pruebas de control de calidad dentro de las tolerancias establecidas, particularmente para las pruebas asociadas al rendimiento del tubo de rayos $\mathrm{X}$ y a la exactitud y repetibilidad del $\mathrm{kV}$ para evitar errores sistemáticos en el cálculo de las magnitudes dosimétricas.

Otras aplicaciones de análisis de espectros no son del todo aptas para ser utilizados en contextos clínicos, por ejemplo, SPEKTR 3.0 no contiene factores de retro dispersión, ni la posibilidad de modificar los diferentes parámetros de exposición sin la necesidad de manejar el lenguaje de programación de Matlab para obtener resultados similares a Xpektrin. Otra limitación de SPEKTR 3.0 es el requerimiento de una licencia para el uso del software que limita el acceso general. SpekCalc ${ }^{(28)}$, otra aplicación de análisis de espectros basada en el modelo semiempírico de Poludniowski(29,30), también requiere de una licencia para acceder a su versión completa. Mención especial por su valor pedagógico tiene la dirección web proporcionada por Siemens donde es posible generar espectros para radiología simple y mamografía, basado en el modelo TASMIPS ${ }^{(31)}$.

El hecho de que Microsoft Excel esté disponible en gran parte de los ordenadores de uso personal y que Xpektrin no requiera de instalación especial alguna más que la habilitación de Macros en la configuración de seguridad del archivo aumenta en gran medida la accesibilidad a la aplicación.

En un contexto pedagógico, los softwares de análisis de espectros mencionados no permiten al usuario acceder en profundidad a los cálculos dosimétricos, por lo constituyen verdaderas cajas negras. Xpektrin contiene el detalle del cálculo dosimétrico en tablas accesibles al usuario, permitiendo una mejor comprensión de los conceptos. Adicionalmente Xpektrin puede ser utilizado como una herramienta didáctica para estudiar el comportamiento del haz bajo diferentes condiciones de exposición, potenciando el auto aprendizaje. Por otro lado, como el acceso a dosímetros y a equipos radiológicos es limitado para las instituciones educacionales, Xpektrin representa una excelente alternativa para complementar o reemplazar el uso de estos instrumentos.

\section{Conclusión}

Xpektrin es una herramienta accesible y de fácil uso que permite determinar magnitudes dosimétricas con buena precisión en diferentes escenarios de exposición. La aplicación requiere de mediciones de rendimiento de tubo y de una o más mediciones de HVL, las que pueden ser obtenidas de controles de calidad rutinarios.

La utilidad de Xpektrin radica en la determinación de dosis a los pacientes, lo que puede utilizarse como apoyo tanto para un sistema de registro dosimétrico como para la determinación de valores típicos de niveles de referencia para diagnóstico de una institución. Adicionalmente tiene un gran valor pedagógico, pudiendo ser utilizado en cursos donde se traten temáticas de técnica radiológica o de física de radiaciones. Los interesados en conseguir el archivo Excel Xpektrin, pueden escribir al correo cdelabarra@hcuch.cl.

\section{Conflicto de intereses}

Los autores declaran no tener ningún conflicto de intereses.

\section{Agradecimientos}

Los autores agradecen a John M. Boone por la facilitación de la planilla de cálculo de los espectros TASMICS.

\section{Referencias}

1. International Atomic Energy Agency. Dosimetry in Diagnostic Radiology: An International Code of Practice, Technical Reports Series No. 457. Viena: IAEA; 2007.

2. Sociedad Española de Radiología Médica. Protoloco Español de Control de Calidad en Radiodiagnóstico. Senda Editorial S.A., editor. Madrid; 2011.

3. Vañó E, Miller DL, Martin CJ, Rehani MM, Kang K, Rosenstein M, et al. ICRP Publication 135: Diagnostic Reference Levels in Medical Imaging. Ann ICRP. 2017;

4. Ayala R, Linares R, García R. MIQuaELa program for the calculation of the DQE in digital radiography. Rev Física Médica. 2009; 10(2): 123-126.

5. Yao Y, Wang AS, Pelc NJ. Efficacy of fixed filtration for rapid kVp-switching dual energy x-ray systems: experimental verification. In: Medical Imaging 2012: Physics of Medical Imaging. SPIE; 2012; 83131F.

6. Perks TD, Dendere R, Irving B, Hartley T, Scholtz P, Lawson $A$, et al. Filtration to reduce paediatric dose for a linear slot-scanning digital x-raymachine. Radiat Prot Dosimetry. 2015; 167(4): 552-561.

7. Pasciak AS, Jones AK, Wagner LK. Application of the 
diagnostic radiological index of protection to protective garments. Med Phys. 2015; 42(2): 653-662.

8. Boone JM, Seibert JA. An accurate method for computer-generating tungsten anode x-ray spectra from 30 to 140 kV. Med Phys. 1997; 24(11): 1661-1670.

9. Boone JM, Fewell TR, Jennings RJ. Molybdenum, rhodium, and tungsten anode spectral models using interpolating polynomials with application to mammography. Med Phys. 1997; 24(12): 1863-1874.

10. Hernandez AM, Boone JM. Tungsten anode spectral model using interpolating cubic splines: Unfiltered x-ray spectra from $20 \mathrm{kV}$ to $640 \mathrm{kV}$. Med Phys. 2014; 41(4): 042101.

11. Seltzer SM, Bartlett DT, Burns DT, Dietze G, Menzel $\mathrm{HG}$, Paretzke HG, et al. Fundamental quantities and units for ionizing radiation. J ICRU. 2011; 11(1): 1-31.

12. Andreo P, Burns DT, Nahum AE, Seuntjens J, Attix $\mathrm{FH}$. Fundamentals of Ionizing Radiation Dosimetry. Wiley; 2017.

13. Hubbell JH, Seltzer SM. Tables of X-Ray mass attenuation coefficients and mass energy-absorption coefficients (version 1.4). NIST. 2004.

14. Benmakhlouf $\mathrm{H}$, Bouchard $\mathrm{H}$, Fransson $\mathrm{A}$, Andreo $P$. Backscatter factors and mass energy-absorption coefficient ratios for diagnostic radiology dosimetry. Phys Med Biol. 2011; 56(22): 7179-7204.

15. Benmakhlouf $H$, Fransson $A$, Andreo P. Influence of phantom thickness and material on the backscatter factors for diagnostic x-ray beam dosimetry. Phys Med Biol. 2013; 58; 247-260.

16. Johns HE, Cunningham JR. The Physics of Radiology. 4th ed. Springfield, Illinois: IL: Thomas; 1983.

17. Punnoose J, Xu J, Sisniega A, Zbijewski W, Siewerdsen JH. Technical Note: spektr 3.0-A computational tool for x-ray spectrum modeling and analysis. Med Phys [Internet]. 2016 Aug 21; 43(8): 4711-4717. Available from: http://www.ncbi.nlm.nih.gov/pmc/articles/ PMC4958109/

18. Bushberg JT. The Essential Physics of Medical Imaging. 3rd ed. Philadelphia: Wolters Kluwer Health/ Lippincott Williams and Wilkins; 2012.

19. Poirier Y, Kouznetsov A, Tambasco M. A simplified approach to characterizing a kilovoltage source spectrum for accurate dose computation. Med Phys. 2012; 39(6): 3041-3050.

20. Organismo internacional de energía atómica. Esta- blecimiento de Niveles Orientativos en Radiografía General y Mamografía, TECDOC-1646. Viena: IAEA; 2010.

21. European Commission. Guidance on diagnostic reference levels (DRLs) for medical exposures: Radiation Protection 109. Radiation Protection Dosimetry. 1998.

22. European Commission. Diagnostic Reference Levels in Thirty-six European Countries. Radiation Protection 180; 2014.

23. Aroua A, Besançon A, Buchillier-Decka I, Trueb P, Valley JF, Verdun FR, et al. Adult reference levels in diagnostic and interventional radiology for temporary use in Switzerland. Radiat Prot Dosimetry. 2004; 111(3): 289-295.

24. Compagnone G, Pagan L, Bergamini C. Local diagnostic reference levels in standard $\mathrm{x}$-ray examinations. Radiat Prot Dosimetry. 2005; 113(1): 54-63.

25. Hart D, Hillier MC, Wall BF. National reference doses for common radiographic, fluoroscopic and dental X-ray examinations in the UK. Br J Radiol. 2009; 82(973): $1-12$.

26. Johnston DA, Brennan PC. Reference dose levels for patients undergoing common diagnostic X-ray examinations in Irish hospitals. Br J Radiol. 2000; 73(868): 396-402.

27. Roch $P$, Aubert $B$. French diagnostic reference levels in diagnostic radiology, computed tomography and nuclear medicine: 2004-2008 Review. Radiat Prot Dosimetry. 2013; 154(1): 52-75.

28. Poludniowski G, Landry G, Deblois F, Evans PM, Verhaegen F. SpekCalc: A program to calculate photon spectra from tungsten anode x-ray tubes. Phys Med Biol. 2009; 54(9): N433.8.

29. Poludniowski GG, Evans PM. Calculation of x-ray spectra emerging from an $x$-ray tube. Part I. Electron penetration characteristics in x-ray targets. Med Phys. 2007; 34(6): 2164-2174.

30. Poludniowski GG. Calculation of x-ray spectra emerging from an $x$-ray tube. Part II. X-ray production and filtration in x-ray targets. Med Phys. 2007; 34(6): 2175-2186.

31. Bätz L. Simulation of X-ray Spectra. Online tool for the simulation of X-ray Spectra [Internet]. Siemens Healthcare $\mathrm{Gm}$

bH. 2019 (cited 2018 dec 20). Available from: https://www. oem-xray-components.siemens.com/x-ray-spectrasimulation\# 